\title{
Performance of six functionals (LDA, PBE, PBESOL, B3LYP, PBE0 and WC1LYP) in the simulation of vibrational and dielectric properties of crystalline compounds. The case of forsterite $\mathrm{Mg}_{2} \mathrm{SiO}_{4}$
}

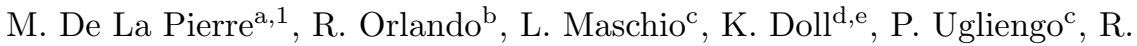 \\ Dovesic $^{\mathrm{c}}$ \\ ${ }^{a}$ Dipartimento di Fisica Sperimentale, Università di Torino and NIS -Nanostructured \\ Interfaces and Surfaces - Centre of Excellence, http://www.nis.unito.it, Via P. Giuria 7, \\ 10125 Torino, Italy \\ ${ }^{b}$ Dipartimento di Scienze e Tecnologie Avanzate, Università del Piemonte Orientale, Via \\ Bellini 25/G, 15100 Alessandria, Italy \\ ${ }^{c}$ Dipartimento di Chimica IFM, Università di Torino and NIS -Nanostructured Interfaces \\ and Surfaces - Centre of Excellence, http://www.nis.unito.it, Via P. Giuria 7, 10125 \\ Torino, Italy \\ ${ }^{d}$ Max-Planck-Institut für Festkörperforschung, Heisenbergstraße 1, D-70569 Stuttgart, \\ Germany \\ ${ }^{e}$ Institut für Mathematische Physik, TU Braunschweig, Mendelssohnstraße 3, D-38106 \\ Braunschweig, Germany
}

\begin{abstract}
The performance of six different density functionals (LDA, PBE, PBESOL, B3LYP, PBE0 and WC1LYP) in describing the infrared spectrum of forsterite, a crystalline periodic system with orthorhombic unit cell (28 atoms in the primitive cell, Pbmn space group), is investigated by using the periodic ab initio CRYSTAL09 code and an all-electron gaussian-type basis set. The transverse optical (TO) branches of the 35 IR active modes are evaluated at the equilibrium geometry together with the oscillator strengths and the high frequency dielectric tensor $\epsilon_{\infty}$. These ingredients permit to compute the dielectric function $\epsilon(\nu)$, and then the reflectance spectrum $R(\nu)$, which is compared with experiment. It turns out that hybrid functionals perform better than LDA and GGA, in general; that B3LYP overperforms WC1LYP and, in turn, PBE0; that PBESOL is better than PBE; that LDA is the worst performing functional among the six
\end{abstract}

Email address: marco.delapierre@unito.it (M. De La Pierre) 
under study.

\section{Introduction}

In the last decade quantum-mechanical methods have been shown to be capable of accurate prediction of many properties of crystalline compounds, including equilibrium geometries and vibrational frequencies.

One of the crucial ingredients of the simulation is the adopted hamiltonian. The opinion that LDA and GGA perform quite well for any crystalline system is widespread in the solid state community $[1,2,3,4]$. In the last years, however, it has been shown that a fraction of Hartree-Fock (HF) exchange improves the description of many properties of solids substantially $[5,6,7,8,9,10,11,12$, $13,14,15]$, so that very recently "hybrid" functionals have been implemented in many periodic codes $[15,16,17,18]$. It is at the same time commonly accepted that B3LYP $[19,20,21]$, the most popular "hybrid" functional in the quantumchemistry molecular community, is not suitable for the description of crystalline solids $[15,22]$. While this is certainly true for metallic systems, experience shows that B3LYP can provide excellent results for many properties, and in particular for the vibrational spectra of insulating systems, such as calcite [11], quartz [23], katoite [24], garnets [8, 10, 25, 26, 27, 28, 29, 30,31].

So far, a detailed comparison of various exchange-correlation (XC) functionals for crystalline systems is limited to simple ionic, covalent and metallic solids with one or two atoms in the unit cell $[1,2,3,4]$. A similar comparison for more complex crystalline systems, or properties other than binding energy, lattice parameter, bulk modulus and band gap is still unavailable.

In the present work we perform an accurate comparison of simulated and experimental infrared (IR) data (wavenumbers and intensities), and of the reflectance spectrum $R(\nu)$, that is the primary information from which experimentalists extract IR wavenumbers and intensities. Equilibrium geometries and dielectric properties are also included in the comparison.

$R(\nu)$ can be simulated by combining different ingredients available in the 
CRYSTAL09 periodic ab initio code, which uses a gaussian-type basis set: (i) the Coupled Perturbed Kohn-Sham scheme (CPKS), recently implemented, is used for the accurate calculation of the high frequency dielectric tensor $\epsilon_{\infty}$ $[32,33,34]$, contributing to the frequency dependent dielectric function $\epsilon(\nu)$; (ii) Born charges, the key quantities for the evaluation of IR intensity, are evaluated through a Berry phase approach [35, 36, 37]; (iii) vibrational modes are obtained by diagonalizing the hessian matrix, whose elements are the second derivatives of the total energy with respect to the atomic cartesian coordinates; the latter are evaluated numerically from analytical gradients [5, 23].

Olivines $\left(\mathrm{X}_{2}^{\mathrm{II}} \mathrm{SiO}_{4}\right)$, due to their relatively large unit cell (28 atoms) and high symmetry, and thanks to the availability of excellent experimental data $[38,39,40,41]$, represent a challenging test for a modern periodic quantummechanical code. Forsterite is one of the end-members of the olivine family, with the $\mathrm{Mg}^{2+}$ cation in the $X$ position. Previous theoretical studies on this compound concern both Raman [42] and infrared spectra, the latter both for pure [43] and H-substituted systems [44, 45, 46], the adopted techniques being molecular dynamics and/or density functional theory.

In a previous preliminary investigation of the IR spectrum of forsterite [47], the B3LYP $[19,20,21]$ hybrid functional was adopted. The same functional was adopted for the study of thermophysical properties of $\mathrm{Mg}_{2} \mathrm{SiO}_{4}$ polymorphs [48]. In this work we extend the analysis to five other functionals, namely to Local Density Approximation (LDA), early (PBE [49]) and newly (PBEsol [1], developed for solids) Generalized Gradient Approximation functionals (GGA), and two other hybrid functionals, namely PBE0 [50] (also known as PBE1PBE or PBEh) and WC1LYP [51]. The availability of very high quality experimental reflection spectra $R(\nu)$ [40], collected by the Japanese infrared beamline of the synchrotron radiation facility SPring8, provides a quite reliable set of data for the comparison of the quality of the results provided by the six functionals. These data, which were published just after our preliminary study [47], are not limited to the peak positions, but also include oscillator strengths, high frequency dielectric tensor and the reflectance spectrum $R(\nu)$. This large set of 
data permits a very accurate comparison between experiment and simulation, and particularly between various flavors of the density functional theory.

\section{Computational details}

Calculations have been performed with the CRYSTAL09 [52] code.

An all-electron basis set is used for all atoms. Silicon, oxygen and magnesium are described by $(8 s)-(6311 s p)-(1 d),(8 s)-(411 s p)-(1 d),(8 s)-(511 s p)-(1 d)$ contractions. The exponents (in bohr ${ }^{-2}$ units) of the most diffuse sp shells are 0.32 and $0.13(\mathrm{Si}), 0.59$ and $0.25(\mathrm{O}), 0.68$ and $0.22(\mathrm{Mg})$; the exponents of the single gaussian d shell are $0.6(\mathrm{Si}), 0.5(\mathrm{O}), 0.5(\mathrm{Mg})[51,47]$.

The level of accuracy in evaluating the Coulomb and Hartree-Fock exchange series is controlled by five parameters [52] that have been set to $7,7,7,7,14$. The threshold on the SCF energy was set to $10^{-8}$ hartree for the geometry optimization, and to $10^{-10}$ hartree for the frequency calculations. The reciprocal space was sampled according to a regular sublattice with shrinking factor [52] equal to 6 that corresponds to 64 independent $\mathbf{k}$ points in the irreducible part of the Brillouin zone.

The DFT exchange-correlation contribution is evaluated by numerical integration over the unit cell volume. In CRYSTAL, radial and angular points of the grid are generated through Gauss-Legendre radial quadrature and Lebedev two-dimensional angular point distributions. In the present calculations, a $(75,974)$ grid has been used (XLGRID keyword in the CRYSTAL09 manual [52]), which corresponds to a pruned grid with 75 radial and 974 angular points. Details about the grid generation, number of points in the reciprocal space and their influence on the accuracy and cost of calculation can be found in Refs. 10 and 25 .

The structure was optimized by using the analytical energy gradients with respect to atomic coordinates and unit-cell parameters [53, 54, 55], within a quasi-Newton scheme combined with the BFGS algorithm for Hessian updating $[56,57,58,59]$. Convergence was checked on both gradient components and 
nuclear displacements, for which the default values [52] are chosen. The calculation of the TO vibrational frequencies at the $\Gamma$ point has been performed within the harmonic approximation; the mass-weighted Hessian matrix $\mathrm{W}$ is constructed by numerical differentiation of the analytical gradients with respect to the atomic cartesian coordinates. The calculated (optimized) equilibrium geometry is taken as reference. Details on the calculation of vibrational frequencies can be found in Refs. 5 and 23.

The strength of the $n^{\text {th }}$ oscillator, $f_{n}$, is defined as:

$$
f_{n, i j}=\frac{4 \pi}{\Omega} \frac{\bar{Z}_{n, i} \bar{Z}_{n, j}}{\nu_{n}^{2}}
$$

where $\Omega$ is the unit cell volume, $i$ and $j$ indicate the three Cartesian components, $\nu_{n}$ is the frequency of the $n^{\text {th }}$ mode and

$$
\bar{Z}_{n, i}=\sum_{A, j} \mathbf{t}_{n, A j} Z_{A, i j}^{*} \frac{1}{\sqrt{M_{A}}}
$$

$Z_{A, i j}^{*}$ is the Born effective charge tensor associated with atom $A$, evaluated through a Berry phase approach $[35,36,37] ; \mathbf{t}_{n, A i}$ is an element of the eigenvectors matrix $\mathrm{T}$ of the mass-weighted Hessian matrix $\mathrm{W}$, that transforms the cartesian atomic directions into the $n^{\text {th }}$ normal coordinate directions; $M_{A}$ is the mass of atom $A$.

The ionic components to the static dielectric tensor $\epsilon_{0, i j}$ are evaluated as the sum of the oscillator strengths: $F_{i j}=\sum_{n} f_{n, i j}$. The electronic high frequency components $\epsilon_{\infty, i j}$ are calculated through the Coupled-Perturbed KS/HF (KohnSham/Hartree-Fock) scheme ([32, 33, 34, 60, 61]).

Manipulation and visualization of structures have been performed with the Jmol 3D engine [62, 63]. Molecular drawings have been rendered with the Inkscape program [64] using input files prepared with Jmol.

\subsection{The reflectance spectrum}

The reflectance spectrum $R(\nu)$ is defined as follows [65]:

$$
R(\nu)=\left|\frac{\sqrt{\epsilon(\nu)-\sin ^{2}(\theta)}-\cos (\theta)}{\sqrt{\epsilon(\nu)-\sin ^{2}(\theta)}+\cos (\theta)}\right|^{2}
$$


where $\theta$ is the incidence angle of the infrared beam; in the present work, we have set $\theta=10^{\circ}$, according to the experimental setup of our reference work [40].

In the case of orthorhombic systems, the complex dielectric function turns out to be a diagonal tensor, with diagonal elements defined as follows:

$$
\epsilon(\nu)_{i i}=\epsilon_{\infty, i i}+\sum_{j} \frac{f_{j, i i} \nu_{j}^{2}}{\nu_{j}^{2}-\nu^{2}-i \nu \gamma_{j}},
$$

where $\nu_{\mathrm{j}}, f_{j}$ and $\gamma_{\mathrm{j}}$ are the TO peak position, the oscillator strength and the damping factor. The electronic high frequency contributions $\epsilon_{\infty, i i}$ are almost constant with respect to frequency in the IR range, as electronic transition energies are very large compared to IR transition energies.

A microscopic approach to the calculation of the dielectric function permits to rewrite Equation 4 as $[66,67,68]$ :

$$
\epsilon(\nu)_{i i}=\epsilon_{\infty, i i}+\frac{4 \pi}{\Omega} \sum_{j} \frac{\bar{Z}_{j, i}^{2}}{\nu_{j}^{2}-\nu^{2}-i \nu \gamma_{j}}
$$

where the definition of the oscillator strength $f_{j, i i}$ according to eq. 1 has been used.

As the harmonic model is used in our simulation, we are unable to compute the $\gamma_{\mathrm{j}}$ damping factors. We used then three different strategies for computing $R^{\text {calc }}(\nu)$ :

(i) the $\gamma_{\mathrm{j}}^{\text {fit }}$ values resulting from the fit of $R^{\text {calc }}(\nu)$ to $R^{\exp }(\nu)$ are used;

(ii) the experimental values $\gamma_{j}^{\exp }$ are used;

(iii) the average $\bar{\gamma}^{\exp }$ of the experimental $\gamma_{j}^{\text {exp }}$ is used, that is $3 \mathrm{~cm}^{-1}$.

The influence of the three choices on the root mean square (RMS) between $R^{\text {calc }}(\nu)$ and $R^{\exp }(\nu)$ will be discussed in the next Section.

From the experimentalists' point of view [40], the digitalized $R^{\exp }(\nu)$ function, through eq. 3 and 4 , provides by best fit the "experimental" values for $\nu_{\mathrm{j}}, f_{j}$ and $\gamma_{\mathrm{j}}$. In the present case, 10,10 and 7 experimental IR peaks were identified for the $x, y$, and $z$ directions, respectively; then the fitting function contains 30, 30 and 21 parameters, respectively.

The comparison among theory and experiment can be performed both at the $R(\nu)$ level, or by comparing calculated and measured $\nu_{\mathrm{j}}$ and $f_{j}$. 


\section{Results}

\subsection{Geometries}

Table 1 refers to the equilibrium geometry, and shows that LDA underestimates the three lattice parameters with respect to the liquid nitrogen experimental datum [69] by $-0.4,-1.5$ and $-1.3 \%$ respectively, whereas PBE overestimates them by about the same amounts, $+1.2,+1.0$ and $+0.9 \%$. B3LYP overestimates the three parameters by less than $1.0 \%$. PBE0, WC1LYP and PBESOL provide by far the best geometry with percentage errors smaller than $0.5 \%$.

\subsection{Infrared modes: wavenumbers and intensities}

Forsterite, as all olivines, is orthorhombic (space group Pbnm, n. 62). There are 28 atoms (four formula units) in the unit-cell, 6 of which symmetry independent, giving rise to 84 vibrational modes. Symmetry analysis shows that:

$$
\Gamma_{\text {total }}=11 A_{g} \oplus 11 B_{1 g} \oplus 7 B_{2 g} \oplus 7 B_{3 g} \oplus 10 A_{u} \oplus 10 B_{1 u} \oplus 14 B_{2 u} \oplus 14 B_{3 u}
$$

A total of 35 IR active modes $\left(9 B_{1 u} \oplus 13 B_{2 u} \oplus 13 B_{3 u}\right)$ and 36 Raman active modes $\left(11 A_{g} \oplus 11 B_{1 g} \oplus 7 B_{2 g} \oplus 7 B_{3 g}\right)$ is then expected, plus $10 A_{u}$ inactive modes. Three additional $B_{1 u}, B_{2 u}$ and $B_{3 u}$ modes correspond to rigid translations.

The calculated and experimental [40] IR-active TO modes are shown in Table 2; in the last four lines statistical indices resulting from the comparison of the various functionals with the experimental data are given. It turns out that B3LYP overperforms the other functionals, featuring a mean absolute error $\overline{|\Delta \nu|}$ as small as $4.7 \mathrm{~cm}^{-1}$, to be compared to 14.3 (LDA), 12.4 (PBE), 8.4 (PBESOL), 12.3 (PBE0) and 8.1 (WC1LYP).

The B3LYP good agreement is probably a combination of two factors, the better quality of the hybrid functionals with respect to LDA and GGA, in particular in describing the short range repulsion, and the B3LYP overestimation of the lattice parameters, that compensates the too hard walls of the potential surface expected at the experimental geometry. As a consequence B3LYP frequencies are in all cases very close to experiment; only in two cases the differences are larger than $10 \mathrm{~cm}^{-1}$ : mode 6 along the $b$ axis $\left(11.8 \mathrm{~cm}^{-1}\right)$, mode 7 along the $c$ axis $\left(10.8 \mathrm{~cm}^{-1}\right)$. 
WC1LYP and PBESOL, besides providing excellent geometries, produce good vibrational frequencies. PBESOL overperforms standard GGA functionals as it recovers the exact condition of the gradient expansion for solids [1]. WC1LYP implements the WC exchange functional, that was specifically designed for solids.

PBE0 provides excellent geometrical data; however, it overestimates the vibrational frequencies systematically $\left(\overline{\Delta \nu}\right.$ is $\left.+12.3 \mathrm{~cm}^{-1}\right)$.

When considering the largest absolute difference, $\left|\Delta \nu_{\max }\right|$, the $|\overline{\Delta \nu}|$ trend is confirmed, with B3LYP being the best performing $\left(\left|\Delta \nu_{\max }\right|=11.8 \mathrm{~cm}^{-1}\right)$, followed by WC1LYP (18.1), PBE0 (21.8) and PBESOL (24.9). LDA $\left(\left|\Delta \nu_{\max }\right|=\right.$ $33.4 \mathrm{~cm}^{-1}$ ) and PBE (39.1) are by far the least performing.

It is interesting to investigate whether $\overline{|\Delta \nu|}$ is a result of many uncorrelated errors for the different modes, or a consequence of systematic errors, such as, for example, the use of a local exchange term, that in turn might produce a wrong geometry. To this aim all calculated frequencies have been shifted by the mean difference $\left(\nu_{i}^{*}=\nu_{i}-\overline{\Delta \nu}\right)$, and the mean absolute difference computed again to give $\overline{|\Delta \nu|}^{*}$ (the star in the table reminds this rigid shift). $\overline{|\Delta \nu|}^{*}$ is around $4 \mathrm{~cm}^{-1}$ for the three hybrid functionals (see table 2), and between 8 and $10 \mathrm{~cm}^{-1}$ for LDA, PBE and PBESOL, providing additional evidence of the intrinsic superiority of hybrids with respect to LDA and GGA.

As regards the oscillator strengths (OS), we can start the analysis by looking at the integrated quantity $F=\sum_{j} f_{j}$, shown in the lowest part of Table 3 . The agreement with experiment is in general satisfactory for all functionals, the differences ranging from 0.8 to $8.3 \%$ for the $a$ axis, from 0.6 to $13.5 \%$ for the $b$ axis, and from 2.5 to $10.3 \%$ for the $c$ axis. The difference between computed and experimental $\Sigma$, that is the sum of $F$ values along $a, b$ and $c$, is as large as -1.37 for LDA, drops to -0.64 for PBESOL and to -0.49 for PBE0; it is minimum for PBE and the two hybrids B3LYP and WC1LYP (0.32, 0.31 and -0.30 , respectively).

The sum of the absolute differences for each direction, $\Delta F=\sum_{j}\left|\Delta f_{j}\right|$, and their sum $\Delta \Sigma$ (the overall sum extends to 31 peaks), are also given in Table 
3. For all functionals but LDA, $\Delta F$ for the $c$ direction is much larger than for $a$ and $b$, possibly indicating problems on the experimental side, as there is no reason for a different performance of the simulation for the different directions. $\Delta \Sigma$ is as large as 5.84 for LDA ( $46.2 \%$ of $\left.\Sigma^{e x p}\right)$; it slightly reduces for PBESOL (5.12), then drops below 3 for the four other functionals. The best performing is WC1LYP $\left(2.19 ; 17.3 \%\right.$ of $\left.\Sigma^{e x p}\right)$. The largest individual difference $\left|\Delta f_{\max }\right|$ confirms that LDA and PBESOL are the worst performing.

A careful analysis shows that quite large contributions to $\Delta F$ come from modes that are very close in frequency (see lines grouped in the $f$ columns of Table 3). This suggests that the one-to-one correspondence between calculated and experimental peaks, performed, within a given symmetry, on the basis of the sequential order of the frequencies, might be wrong when peaks are very close to each other. A second source of discrepancy is the following: when modes are close in frequency (and then the corresponding peaks overlap), the deconvolution of the experimental spectrum through a best fit process can be affected by large errors, due to the strong correlation between the parameters describing the intensity of these peaks. This is evident in the couples of modes $(5,6)$ of the $a$ and $c$ axis: $\Delta f$ for one peak is large and positive; for the other large and negative, so that their sum becomes very small. For this reason, $f$ belonging to two (or more) frequencies with $\Delta \nu \leq 10 \mathrm{~cm}^{-1}$, or with $\Delta \nu \leq 30 \mathrm{~cm}^{-1}$ and $f_{\text {calc }} \geq 0.5$ were grouped before comparison among theory and experiment. This strategy reduces the absolute difference $\Delta \Sigma$, (and $\left|\Delta f_{\max }\right|$ as well), that for WC1LYP is now 1.39 (11.0\% of $\left.\Sigma^{e x p}\right)$ and slightly larger for PBE, PBE0 and B3LYP (from 1.51 to 1.71), whereas it remains quite large for PBESOL and LDA.

\subsection{The $R(\nu)$ reflectance spectrum}

As anticipated in Section 2.1, the digitalized reflectance spectrum $R^{\exp }(\nu)$ has been used by experimentalists [40] to obtain by best fit the "experimental" frequencies $\nu_{j}^{\text {exp }}$, intensities $f_{j}^{\text {exp }}$ and damping factors $\gamma_{j}^{\text {exp }}$ of the observed peaks (see eq. 3 and 4 ; we remind that with this procedure only a fraction of 
the expected peaks is identified). The quality of this fit can be estimated by recomputing the "experimental fitted" reflectance spectrum $R^{f i t}(\nu)$ with these data. The root mean square RMS deviation between $R^{f i t}(\nu)$ and $R^{\exp }(\nu)$ is reported in the last column of Table 4; the corresponding curve is shown in the last panel of Figure 2, in the case of the $a$ axis. We notice the very good agreement between the two spectra, in spite of the fact that 2, 1 and 1 modes are missing for the three axes, respectively (see the lists of $\nu^{\exp }$ and $f^{\exp }$ in Tables 2 and 3); this is not surprising, as it has been shown in Section 3.2 that these modes feature very low calculated intensities. The low value of $\mathrm{RMS}^{\text {fit }}$ also indicates that the model described through eq. 3 and 4 is adequate.

As regards simulation, $\epsilon_{\infty}$ ( see eq. 4) has been evaluated through the CPHF scheme. Table 5 shows that hybrid functionals underestimate by about $9 \%$ the experiment, whereas LDA and PBE come very close to it.

In order to build-up our "computed" reflectance curve $R^{\text {calc }}(\nu)$, besides the calculated $\epsilon_{\infty}, \nu_{j}$ and $f_{j}$, we also require the $\gamma_{j}$ damping factors. As anticipated in Section 2.1, our harmonic model does not provide them, so that we must rely on the experimental data. Three different $\gamma_{j}$ values have been used, the aim being to avoid that a particular choice could bias the analysis of the relative performance of the six functionals. The simulated spectrum has been generated by using the $\gamma_{j}^{e x p}$ values mentioned above, or the mean value of these $\left(\bar{\gamma}^{\exp }\right.$, equal to $3 \mathrm{~cm}^{-1}$ ), or $\gamma_{j}^{\text {fit }}$, obtained by best fit between the digitalized reflectance spectrum $R^{\exp }(\nu)$ and $R^{\text {calc }}(\nu)$ itself, the latter containing $\gamma_{j}^{f i t}$ as parameters. The effect of the choice of the damping factor on the reflectance curve is shown graphically in the top panel of Figure 1, in the case of the $a$ axis and the B3LYP functional.

Table 4, that provides the RMS deviation between the various $R^{\text {calc }}(\nu)$ curves and $R^{\exp }(\nu)$, shows that:

a) for a given choice of $\gamma_{j}$ and functional, the RMS deviation is not very different for the three directions, so that the mean value $\overline{R M S}$ can be used for the comparison among functionals.

b) The differences among the $\overline{R M S}$ obtained with the three choices for $\gamma_{j}$ are 
not very large, so that the relative merits of the various functionals are essentially the same, independently from the choice of $\gamma_{j}$ (see also Refs. 30, 31).

c) B3LYP and PBESOL provide the smallest $\overline{R M S}$, followed by WC1LYP. On the other hand, PBE, PBE0 and LDA provide about the same and larger $\overline{R M S}$ values.

Examples of $R^{\text {calc }}(\nu)$ curves computed with different $\gamma_{j}$ choices, for the three crystallographic axes and with the various adopted functionals are given in Figures 1 and 2.

\section{Conclusions}

The vibrational properties and the reflectance IR spectrum of forsterite have been calculated at an $a b$ initio quantum-mechanical level with six different hamiltonians, and compared with the available highly accurate experimental data.

The large set of data makes the comparison among the functionals difficult, because the relative performance varies with the considered property. For this reason we summarized the most relevant statistical indices discussed in the previous Section in a single table (see Table 6): the percentage error on the volume $(\Delta V)$, the mean absolute difference between calculated and experimental wavenumbers $(\overline{|\Delta \nu|})$, the equivalent quantity for the oscillator strengths $(\Delta \Sigma$ "after grouping", see data in parentheses in Table 3), the difference among the integrated oscillator strengths $\left(\sigma=\Sigma_{\text {calc }}-\Sigma_{\text {exp }}\right)$ and the root mean square among reflection spectra $(\overline{R M S})$.

The agreement is excellent when the B3LYP functional is used, with respect to most of the studied properties: vibrational frequencies in particular, oscillator strengths, reflectance curves, whereas the volume is slightly too large. WC1LYP performs very well, too, for all properties; the same is true for PBESOL, with the exception of the IR intensities and related properties. WC1LYP, PBESOL and PBE0 provide better geometries than B3LYP; PBE0 yields good oscillator strengths, but poorer wavenumbers than B3LYP. LDA and PBE provide the worst geometries, poor vibrational frequencies; LDA intensities are the worst 
ones determined, whereas the PBE ones are in line with the results from hybrid functionals. As regards the $\mathrm{R}(\nu)$ spectrum, LDA, PBE and PBE0 are the worst performing.

Overall, then, hybrid functionals for the present system perform better than GGA, and B3LYP and WC1LYP better than PBE0. For particular properties PBE or PBESOL can be competitive. LDA is the worst in all cases.

The present study highlights the relevance of either the exact exchange contribution (B3LYP, WC1LYP, PBE0) or a specific design for the solid state (PBESOL, WC).

These results confirm previous studies [8, 10, 25, 27, 28, 29, 30, 31, 70, 71, 72] referring to a large family of silicates, including hydroxides, showing that B3LYP produces in general excellent vibrational spectra.

\section{Acknowledgements}

The authors wish to thank Raffaella Demichelis for her contribution to the calculations. 


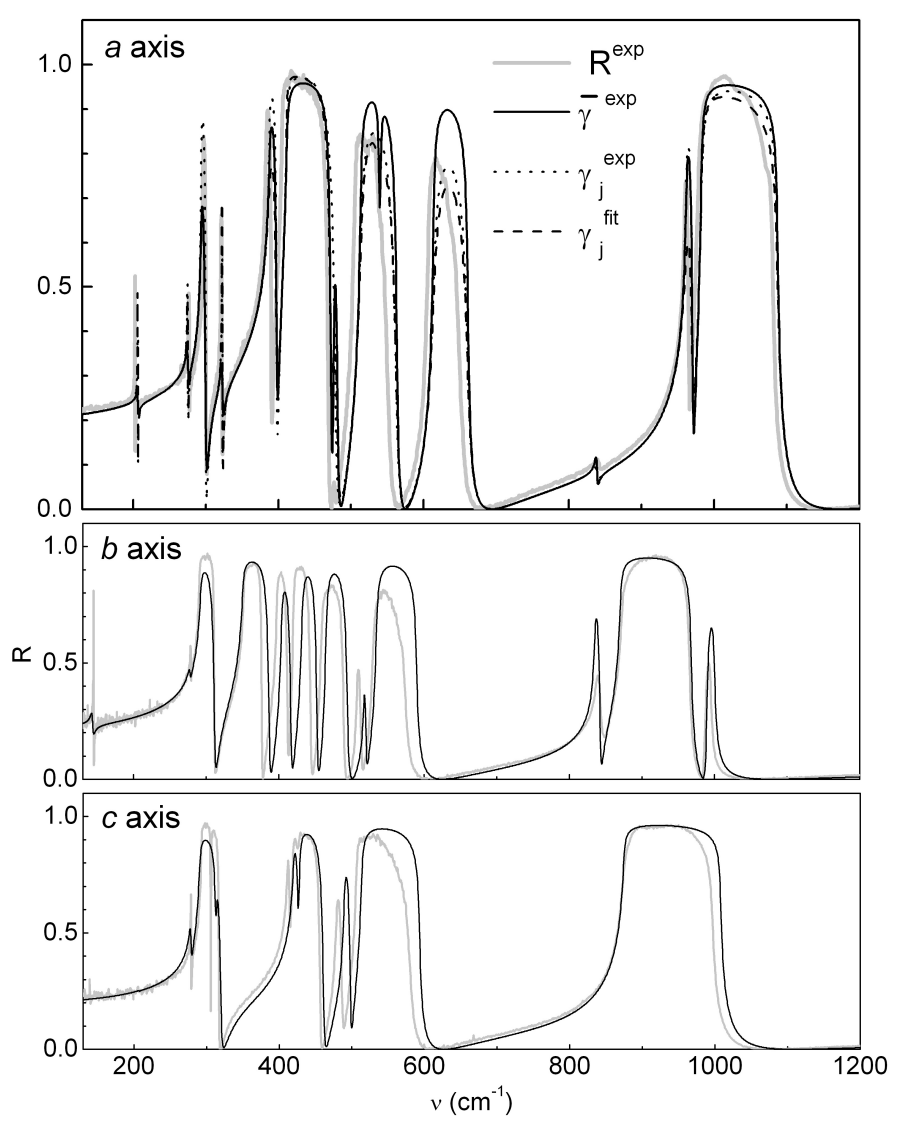

Figure 1: Reflectance spectrum $R(\nu)$ along the three axes of forsterite, computed at the B3LYP level. In the case of $a$ axis, different sets of damping factors $\gamma_{j}$ have been used: $\gamma_{j}^{f i t}$ (dashed line), $\gamma_{j}^{e x p}$ (dotted line), $\bar{\gamma}^{\exp }$ (solid line); see caption to Table 4 for further details. Experimental $R^{\exp }(\nu)$ from Ref. 40 is also shown (thick gray solid line), for sake of comparison. In the case of $b$ and $c$ axes, only the experimental [40] and the $\bar{\gamma}^{e x p}$ calculated curves are reported. 


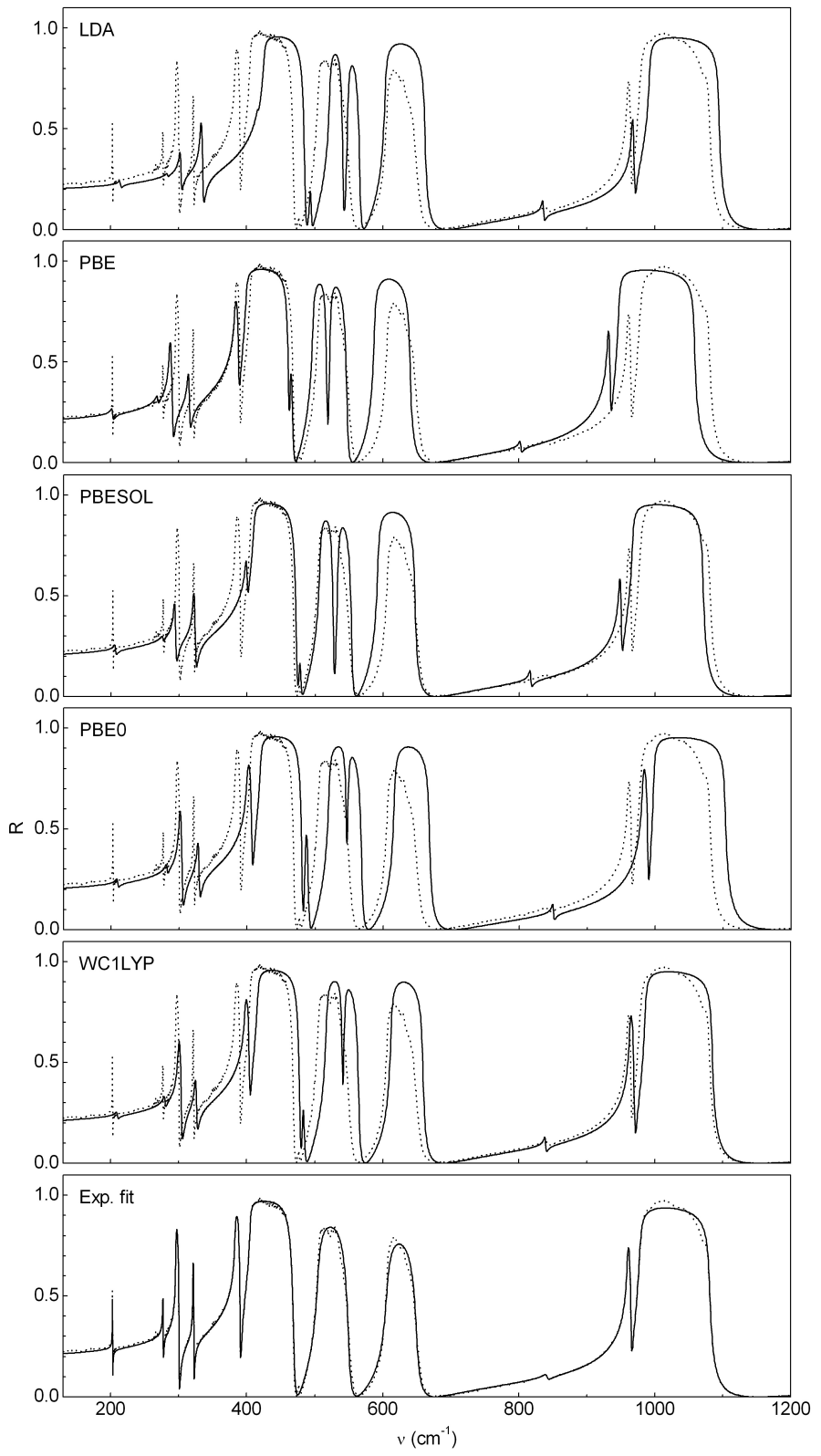

Figure 2: Calculated ( $\bar{\gamma}^{e x p}$, continuous line) and experimental (dotted line) [40] reflectance spectrum $R(\nu)$ along the $a$-axis of forsterite. In the last box the fitted and instrumental experimental spectra, both from Ref. 40, are compared. 


\begin{tabular}{|c|c|c|c|c|c|c|c|}
\hline & LDA & PBE & PBESOL & B3LYP & PBEO & WC1LYP & Exp. ${ }^{a}$ \\
\hline $\mathrm{a}$ & $4.726(-0.4)$ & $4.804(+1.2)$ & $4.769(+0.5)$ & $4.789(+0.9)$ & $4.760(+0.3)$ & $4.771(+0.5)$ & 4.746 \\
\hline $\mathrm{b}$ & $10.030(-1.5)$ & $10.280(+1.0)$ & $10.165(-0.1)$ & $10.254(+0.7)$ & $10.175(0.0)$ & $10.183(0.0)$ & 10.18 \\
\hline $\mathrm{c}$ & $5.898(-1.3)$ & $6.032(+0.9)$ & $5.973(0.0)$ & $6.009(+0.6)$ & $5.970(-0.1)$ & $5.974(0.0)$ & 5.976 \\
\hline Vol. & $279.58(-3.2)$ & $297.85(+3.2)$ & $289.55(+0.3)$ & $295.10(+2.2)$ & $289.14(+0.2)$ & $290.23(+0.5)$ & 288.7 \\
\hline $\mathrm{Si}-\mathrm{O}$ & $1.621(+0.3)$ & $1.640(+1.5)$ & $1.633(+1.0)$ & $1.628(+0.8)$ & $1.623(+0.4)$ & $1.626(+0.6)$ & 1.616 \\
\hline $\mathrm{Si}-\mathrm{O}_{n}$ & $1.665(+1.0)$ & $1.685(+2.2$ & $1.677(+1.7)$ & $1.673(+1.4)$ & $1.665(+1.0)$ & $1.670(+1.3)$ & 1.649 \\
\hline $\mathrm{Mg} 1-\mathrm{O} m i$ & $2.043(-1.2)$ & $2.080(+0.5$ & $2.064(-0.2)$ & $2.073(+0.2$ & $2.062(-0.4)$ & $2.064(-0.2$ & 2.069 \\
\hline Mg1-O $\max$ & $2.093(-1.6)$ & $2.136(+0.5$ & $2.117(-0.4)$ & $2.131(+0.2$ & $2.117(-0.4)$ & $2.120(-0.3)$ & 2.126 \\
\hline Mg2- & $2.017(-1.1)$ & $2.065(+1.2$ & $2.042(+0.1)$ & $2.063(+1.1$ & $2.047(+0.3)$ & $2.048(+0.4)$ & 2.040 \\
\hline $\mathrm{Mg} 2-$ & $2.171(-1.7$ & 2.221 & $2.198(-0.5)$ & 2.222 & $2.200(-0.3)$ & $2.208(0.0)$ & 2.208 \\
\hline
\end{tabular}

Table 1: Calculated and experimental geometry of forsterite (Pbnm space group, 28 atoms in the unit cell, 6 of which symmetry independent). $a, b$ and $c$ are the cell parameters. The maximum $(\max )$ and minimum $(\min ) \mathrm{Si}-\mathrm{O}$ and $\mathrm{Mg}-\mathrm{O}$ bond distances are reported. Percentage differences with respect to experimental values are given in parentheses. Distances are in $\AA$, volumes in $\AA^{3}$. ${ }^{a}$ Ref. [69]. 


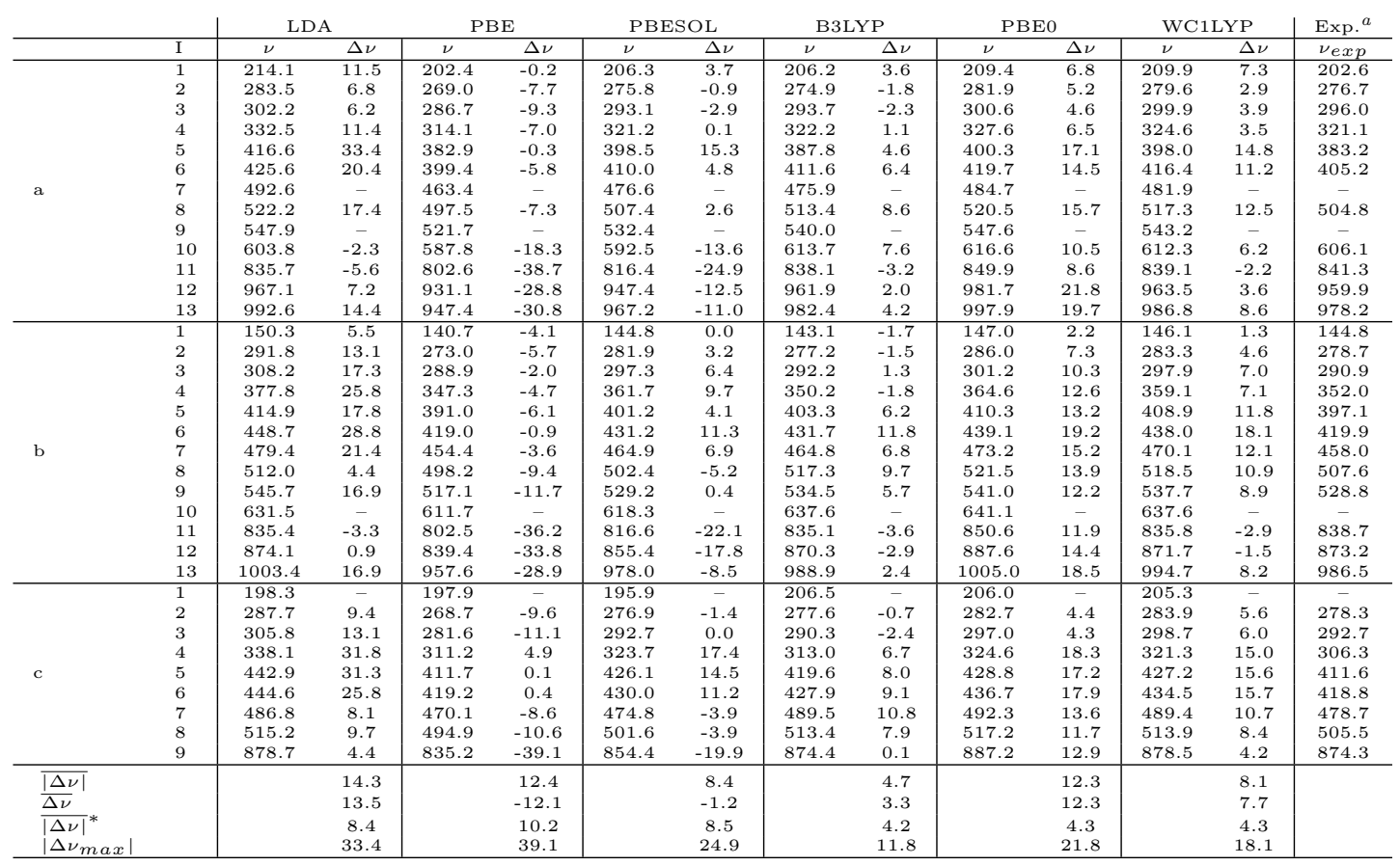

Table 2: Calculated and experimental vibrational frequencies $\nu\left(\mathrm{cm}^{-1}\right)$ of the IR active transverse optical (TO) modes along the three axes of forsterite (featuring $B_{2 u}, B_{3 u}$ and $B_{1 u}$ symmetries, respectively). $\Delta \nu$ is the difference between calculated and experimental quantities. Statistical indices ( $x$ is either $\nu$ or $f$ ): $\overline{|\Delta x|}=\sum_{i=1}^{N}\left|\Delta x_{i}\right| / N$ is the mean absolute difference, $\overline{\Delta x}=\sum_{i=1}^{N} \Delta x_{i} / N$ is the mean difference, $\left|\Delta x_{\text {max }}\right|$ is the maximum absolute difference, $\overline{\mid \Delta x}^{*}=\sum_{i=1}^{N}\left|\Delta x_{i}-\overline{\Delta x}\right| / N$ is the mean absolute difference computed after shifting the frequencies by $\overline{\Delta x} ; N$ is the number of available experimental data, on which statistics are performed; $N=31$, over the three axes. ${ }^{a}$ Ref. [40]. 


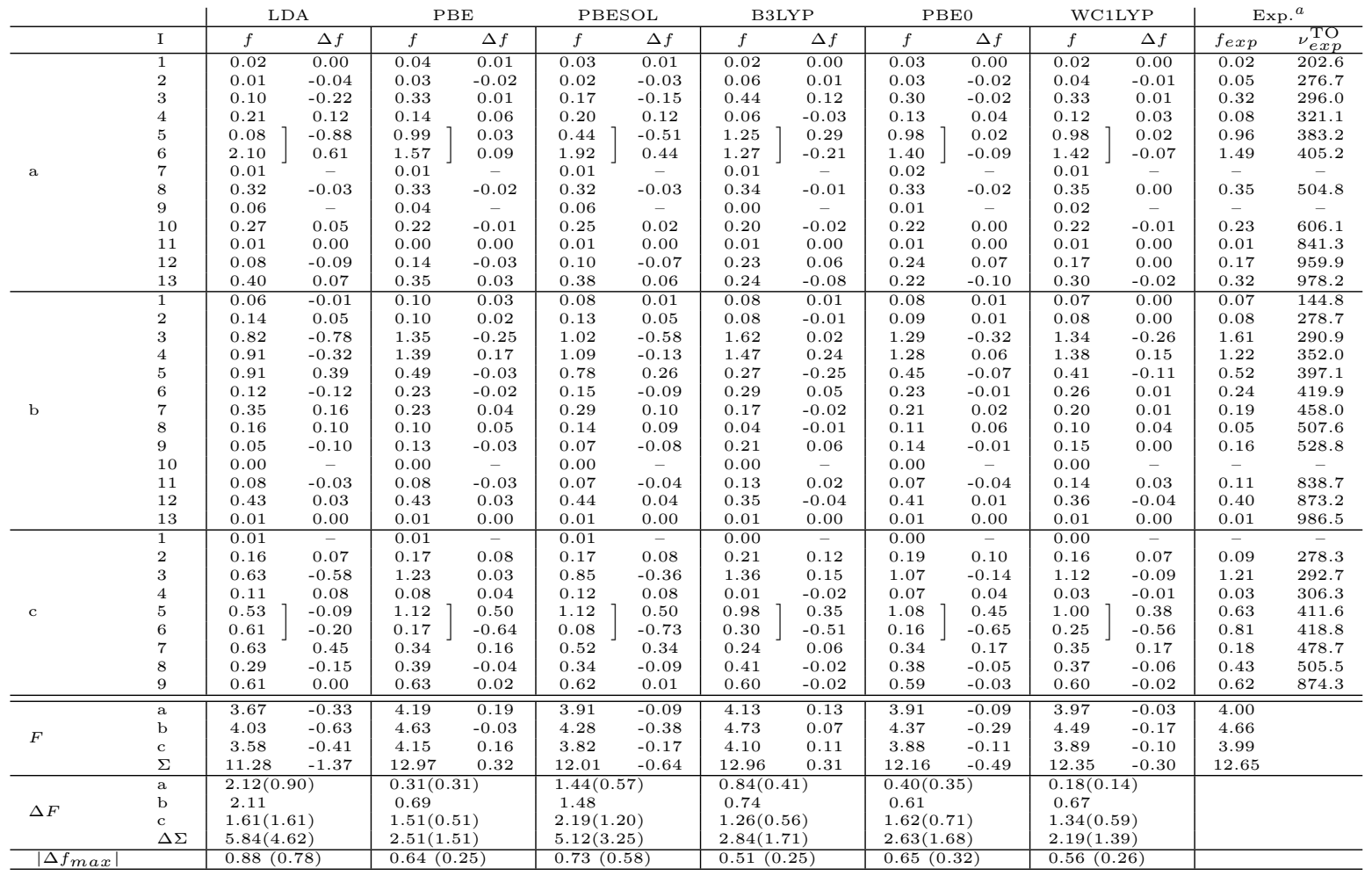

Table 3: Calculated and experimental oscillator strengths $f$ (adimensional) of the IR active transverse optical (TO) modes along the three axes of forsterite. $\Delta f$ is the difference between calculated and experimental quantities. $F=\sum_{j} f_{j}$ is the sum of the oscillator strengths; $\Delta F=\sum_{j}\left|\Delta f_{j}\right|$ is the sum of the absolute differences between calculated and experimental oscillator strengths; these two quantities are computed for each crystallographic axis (a,b,c) and summed over the three axes ( $\Sigma$ and $\Delta \Sigma$, respectively). $\left|\Delta f_{\max }\right|$ is defined as in Table 2; in this case, $N$ is equal to 31 ( 29 for the reduced set with grouped values for $f$ ). Oscillator strengths are grouped either when the corresponding calculated frequencies differ $(\delta \nu)$ by less than $10 \mathrm{~cm}^{-1}$, or when $\delta \nu \leq 30 \mathrm{~cm}^{-1}$ and $f_{\text {calc }} \geq 0.5$; values in parentheses refer to this "amended" set of data. ${ }^{a}$ Ref. [40]. 


\begin{tabular}{|c|c|c|c|c|c|c|c|c|}
\hline & & LDA & PBE & $\operatorname{PBESOL}^{a}$ & B3LYP & PBE0 & $\mathrm{WC}_{1} \mathrm{LYP}^{a}$ & Exp. fit \\
\hline \multirow{3}{*}{$\mathrm{a}$} & $\gamma_{j}^{f}$ & 0.2150 & 0.1757 & 0.1302 & 0.1366 & 0.2340 & 0.1806 & - \\
\hline & & 0.2150 & 0.1757 & 0.1383 & 0.1606 & 0.2411 & 0.1810 & 0.0187 \\
\hline & $\bar{\gamma}^{\exp }$ & 0.2351 & 0.1823 & 0.1465 & 0.1647 & 0.2450 & 0.1837 & - \\
\hline \multirow{3}{*}{ b } & $\gamma_{j}^{f i t}$ & 0.2320 & 0.1830 & 0.1477 & 0.1398 & 0.2419 & 0.1883 & - \\
\hline & 3 & 0.2390 & 0.1914 & 0.1583 & 0.1687 & 0.2600 & 0.2002 & 0.0165 \\
\hline & $\bar{\gamma}^{e x p}$ & 0.2335 & 0.1955 & 0.1600 & 0.1701 & 0.2570 & 0.1952 & - \\
\hline \multirow{3}{*}{ c } & $\gamma_{j}^{f i t}$ & 0.1851 & 0.2146 & 0.1637 & 0.1220 & 0.1713 & 0.1391 & - \\
\hline & $\gamma_{i}^{\text {exp }}$ & 0.1956 & 0.2176 & 0.1691 & 0.1539 & 0.2027 & 0.1454 & 0.0185 \\
\hline & $\bar{\gamma}^{\exp }$ & 0.1927 & 0.2241 & 0.1695 & 0.1466 & 0.2046 & 0.1396 & - \\
\hline \multirow{3}{*}{$\overline{R M S}$} & $\gamma_{j}^{f i t}$ & 0.2107 & 0.1911 & 0.1472 & 0.1328 & 0.2157 & 0.1693 & - \\
\hline & $\gamma_{i}^{\text {exp }}$ & 0.2165 & 0.1949 & 0.1552 & 0.1611 & 0.2346 & 0.1755 & 0.0179 \\
\hline & $\bar{\gamma}^{e x p}$ & 0.2204 & 0.2006 & 0.1587 & 0.1605 & 0.2355 & 0.1728 & - \\
\hline
\end{tabular}

Table 4: Root mean square RMS of the difference between calculated $\left(R^{\text {calc }}(\nu)\right.$ or $\left.R^{\text {fit }}(\nu)\right)$ and experimental $\left(R^{\exp }(\nu)\right)$ reflectance curves for the three crystallographic axes (a,b,c); mean value among the three axes $(\overline{R M S})$. The first six columns refer to $R^{\text {calc }}(\nu)$ computed in the present work; the last column refers to $R^{f i t}(\nu)$ obtained by experimentalists in Ref. 40. $R^{\text {calc }}(\nu)$ is obtained with three different choices for the damping factors: $\gamma_{j}^{f i t}$ : values obtained in the present work by best-fit with respect to $R^{\exp }(\nu) ; \gamma_{j}^{e x p}$ : experimental values from Ref. 40; $\bar{\gamma}^{e x p}$ : average of experimental values $\left(3 \mathrm{~cm}^{-1}\right)$ [40]. The investigated range is $130 \div 1200 \mathrm{~cm}^{-1}$, and includes 2636, 2952 and 2274 points for the $a, b$ and $c$ axes, respectively. ${ }^{a}$ Experimental values [40] of the optical dielectric tensor are used in the case of PBESOL and WC1LYP functionals.

\begin{tabular}{|c|c|c|c|c|c|c|c|c|c|}
\hline & & LDA & $\mathrm{PBE}$ & $\operatorname{PBESOL}^{a}$ & B3LYP & PBE0 & $\mathrm{WC}_{1 \mathrm{LYP}}{ }^{a}$ & Exp. fit $^{b}$ & Exp. ${ }^{c}$ \\
\hline \multirow{3}{*}{$\mathrm{x}$} & $\epsilon_{0}$ & 6.487 & 6.972 & 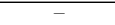 & 6.702 & 6.499 & - & 6.77 & 6.87 \\
\hline & $\epsilon_{\infty}$ & 2.819 & 2.779 & - & 2.571 & 2.590 & - & 2.77 & 2.789 \\
\hline & & 3.669 & 4.193 & 3.910 & 4.131 & 3.909 & 3.966 & 4.00 & 4.08 \\
\hline \multirow{3}{*}{$\mathrm{y}$} & $\epsilon_{0}$ & 6.682 & 7.236 & - & 7.149 & 6.815 & & 7.32 & 7.39 \\
\hline & $\epsilon_{\infty}$ & 2.653 & 2.606 & - & 2.423 & 2.445 & - & 2.66 & 2.673 \\
\hline & $F$ & 4.029 & 4.629 & 4.285 & 4.726 & 4.370 & 4.488 & 4.66 & 4.72 \\
\hline \multirow{3}{*}{$\mathrm{z}$} & $\epsilon_{0}$ & 6.284 & 6.811 & - & 6.577 & 6.377 & - & 6.70 & 6.74 \\
\hline & $\epsilon_{\infty}$ & 2.708 & 2.665 & - & 2.473 & 2.493 & - & 2.71 & 2.726 \\
\hline & $F$ & 3.576 & 4.147 & 3.824 & 4.104 & 3.884 & 3.893 & 3.99 & 4.01 \\
\hline
\end{tabular}

Table 5: Calculated and experimental static dielectric tensor $\left(\epsilon_{0}\right)$ and its components: the electronic high frequency $\left(\epsilon_{\infty}\right)$ and the ionic one, evaluated as the sum of the oscillator strengths $\left(F=\sum_{j} f_{j}\right)$. The three cartesian directions correspond to the crystallographic ones, so that the dielectric tensor turns out to be diagonal. ${ }^{a} \mathrm{CPHF}$ calculation of optical dielectric tensor currently not available for PBESOL and WC1LYP functionals. ${ }^{b}$ Values obtained through a fitting procedure [40]. ${ }^{c}$ Values for $\epsilon_{0}$ from Ref. 73; values for $\epsilon_{\infty}$ from Ref. 74 .

\begin{tabular}{l|cccccc} 
& LDA & PBE & PBESOL & B3LYP & PBE0 & WC1LYP \\
\hline$\Delta V$ & -3.2 & +3.2 & +0.3 & +2.2 & +0.2 & +0.5 \\
$\frac{|\Delta \nu|}{\sigma}$ & 14.3 & 12.4 & 8.4 & 4.7 & 12.3 & 8.1 \\
$\Delta \Sigma$ & -1.37 & 0.32 & -0.64 & 0.31 & -0.49 & -0.30 \\
$\frac{\Delta \Sigma}{R M S}$ & 4.62 & 1.51 & 3.25 & 1.71 & 1.68 & 1.39 \\
\hline
\end{tabular}

Table 6: Summary of the main statistical indices discussed in the present work. $\Delta V\left(\AA^{3}\right)$ is the percentage error on the volume (from Table 1$) ; \overline{|\Delta \nu|}\left(\mathrm{cm}^{-1}\right)$ is the mean absolute difference for the frequencies (Table 2); $\sigma=\Sigma_{\text {calc }}-\Sigma_{\text {exp }}$ (dimensionless) is the difference among integrated oscillator strengths (Table 3); $\Delta \Sigma$ (dimensionless) is the sum of the absolute differences for the oscillator strengths, "after grouping" (data in parentheses in Table 3); $\overline{R M S}$ is the root mean square between calculated (with $\bar{\gamma}^{\exp }$ ) and experimental reflectance spectra $R(\nu)$, averaged among the three axes (Table 4$)$. 
[1] Perdew, J.; Ruzsinsky, A.; Csonka, G. I.; Vydrov, O. A.; Scuseria, G. E.; Constantin, L. A.; Zhou, X.; Burke, K. Phys. Rev. Lett. 2008, 100, 136406.

[2] Mattsson, A. E.; Armiento, R.; Paier, J.; Kresse, G.; Wills, J. M.; Mattsson, T. R. J. Chem. Phys. 2008, 128, 084714.

[3] Tran, F.; Laskowski, R.; Blaha, P.; Schwarz, K. Phys. Rev. B 2007, 75, 115131

[4] Haas, P.; Tran, F.; Blaha, P. Phys. Rev. B 2009, 79, 085104.

[5] Pascale, F.; Zicovich-Wilson, C. M.; Gejo, F. L.; Civalleri, B.; Orlando, R.; Dovesi, R. J. Comp. Chem. 2004, 25, 888-897.

[6] Corà, F.; Alfredsson, M.; Mallia, G.; Middlemiss, D. S.; Mackrodt, W. C.; Dovesi, R.; Orlando, R. The performance of hybrid density functionals in solid state chemistry, Vol. 113, Principles and Applications of Density Functional Theory in Inorganic Chemistry II of Structure and Bonding; Springer Berlin, Heidelberg, 2004.

[7] Bilc, D. I.; Orlando, R.; Rignanese, G. M.; Íñiguez, J.; Ghosez, P. Phys. Rev. B 2008, 77, 165107.

[8] Pascale, F.; Catti, M.; Damin, A.; Orlando, R.; Saunders, V. R.; Dovesi, R. J. Phys. Chem. B 2005, 109, 18522-18527.

[9] Demichelis, R.; Noel, Y.; Civalleri, B.; Roetti, C.; Ferrero, M.; Dovesi, R. J. Phys. Chem. B 2007, 111, 9337-9346.

[10] Pascale, F.; Zicovich-Wilson, C. M.; Orlando, R.; Roetti, C.; Ugliengo, P.; Dovesi, R. J. Phys. Chem. B 2005, 109, 6146-6152.

[11] Prencipe, M.; Pascale, F.; Zicovich-Wilson, C.; Saunders, V. R.; Orlando, R.; Dovesi, R. Phys. Chem. Min. 2004, 31, 559-564.

[12] Heyd, J.; Peralta, J. E.; Scuseria, G.; Martin, R. L. J. Chem. Phys. 2005, 123, 174101. 
[13] Paier, J.; Marsman, M.; Hummer, K.; Kresse, G.; Gerber, I. C.; Angyan, J. G. J. Chem. Phys. 2006, 124, 154709.

[14] Gerber, I. C.; Angyan, J. G.; Marsman, M.; Kresse, G. J. Chem. Phys. 2007, 127, 054101.

[15] Paier, J.; Marsman, M.; Kresse, G. J. Chem. Phys. 2007, 127, 024103.

[16] Kudin, K. N.; Scuseria, G. Phys. Rev. B 2000, 61, 16440-16453.

[17] Hafner, J. J. Comp. Chem. 2008, 29, 2044-2078.

[18] Tran, F.; Blaha, P.; Schwarz, K.; Novak, P. Phys. Rev. B 2006, 74, 155108.

[19] Becke, A. D. J. Chem. Phys. 1993, 98, 5648-5652.

[20] Lee, C.; Yang, W.; Parr, R. G. Phys. Rev. B 1988, 37, 785-789.

[21] Stephens, P.; Devlin, F.; Chabalowski, C.; Frisch, M. J. Phys. Chem. 1994, 98, 11623-11627.

[22] Marsman, M.; Paier, J.; Stroppa, A.; Kresse, G. J. Phys.: Condens. Matter 2008, 20, 064201.

[23] Zicovich-Wilson, C. M.; Pascale, F.; Roetti, C.; Orlando, V. R. S. R.; Dovesi, R. J. Comp. Chem. 2004, 25, 1873-1881.

[24] Orlando, R.; Torres, J.; Pascale, F.; Ugliengo, P.; Wilson, C. Z.; Dovesi, R. J. Phys. Chem. B 2006, 110, 692-701.

[25] Zicovich-Wilson, C. M.; Torres, F. J.; Pascale, F.; Valenzano, L.; Orlando, R.; Dovesi, R. J. Comp. Chem. 2008, 29, 2268-2278.

[26] Dovesi, R.; Valenzano, L.; Pascale, F.; Zicovich-Wilson, C.; Orlando, R. J. Raman Spectrosc. 2009, 40, 416-418.

[27] Valenzano, L.; Meyer, A.; Demichelis, R.; Civalleri, B.; Dovesi, R. Phys. Chem. Min. 2009, 36, 415-420. 
[28] Ferrari, A. M.; Valenzano, L.; Meyer, A.; Orlando, R.; Dovesi, R. J. Phys. Chem. A 2009, 113, 11289-11294.

[29] Valenzano, L.; Pascale, F.; Ferrero, M.; Dovesi, R. Int. J. Quantum Chem. 2010, 110, 416-421.

[30] Dovesi, R.; De La Pierre, M.; Ferrari, A. M.; Pascale, F.; Maschio, L.; Zicovich-Wilson, C. M. in preparation, 2010.

[31] Maschio, L.; Ferrabone, M.; Meyer, A.; Garza, J.; Dovesi, R. submitted to Chem. Phys. Lett., 2010.

[32] Ferrero, M.; Rérat, M.; Orlando, R.; Dovesi, R. J. Comp. Chem. 2008, 29, 1450-1459.

[33] Ferrero, M.; Rérat, M.; Orlando, R.; Dovesi, R. J. Chem. Phys. 2008, 128, 014110 .

[34] Ferrero, M.; Rérat, M.; Kirtman, B.; Dovesi, R. J. Chem. Phys. 2008, 129, 244110.

[35] Dall'Olio, S.; Dovesi, R.; Resta, R. Phys. Rev. B 1997, 56, 10105-10114.

[36] Baranek, P.; Zicovich-Wilson, C.; Roetti, C.; Orlando, R.; Dovesi, R. Phys. Rev. B 2001, 64, 125102.

[37] Noël, Y.; Zicovich-Wilson, C.; Civalleri, B.; D'Arco, P.; Dovesi, R. Phys. Rev. B 2002, 65, 014111.

[38] Kolesov, B.; Geiger, C. Phys. Chem. Min. 2004, 31, 142-154.

[39] Sogawa, H.; Koike, C.; Chihara, H.; Suto, H.; Tachibana, S.; Tsuchiyama, A.; Kozasa, T. A. \& A. 2006, 451, 357-361.

[40] Suto, H.; Sogawa, H.; Tachibana, S.; Koike, C.; Karoji, H.; Tsuchiyama, A.; Chihara, H.; Mizutani, K.; Akedo, J.; Ogiso, K.; Fukui, T.; Ohara, S. Mon. Not. R. Astron. Soc. 2006, 370, 1599-1606. 
[41] Pitman, K. M.; Dijkstra, C.; Hofmeister, A. M.; Speck, A. K. Mon. Not. R. Astron. Soc. 2010, 406, 460-481.

[42] McKeown, D. A.; Bell, M. I.; Caracas, R. Am. Mineral. 2010, 95, 980-986.

[43] Li, L.; Wentzcovitch, R. M.; Weidner, D. J.; Da Silva, C. R. S. J. Geophys. Res.-Sol. Ea. 2007, 112, B05206.

[44] Braithwaite, J.; Wright, K.; Catlow, C. J. Geophys. Res.-Sol. Ea. 2003, $108,2284$.

[45] Walker, A. M.; Demouchy, S.; Wright, K. Eur. J. Miner. 2006, 18, 529543.

[46] Shaw, D. M.; Tse, J. S. Am. Mineral. 2007, 92, 1593-1600.

[47] Noel, Y.; Catti, M.; D’Arco, P.; Dovesi, R. Phys. Chem. Min. 2006, 33, 383.

[48] Ottonello, G.; Civalleri, B.; Ganguly, J.; Zuccolini, M. V.; Nöel, Y. Phys. Chem. Min. 2009, 36, 87-106.

[49] Perdew, J. P.; Burke, K.; Ernzerhof, M. Phys. Rev. Lett. 1996, 77, 38653868 .

[50] Adamo, C.; Barone, V. J. Chem. Phys. 1999, 110, 6158-6170.

[51] Demichelis, R.; Civalleri, B.; Ferrabone, M.; Dovesi, R. Int. J. Quantum Chem. 2010, 110, 406-415.

[52] Dovesi, R.; Saunders, V. R.; Roetti, C.; Orlando, R.; Zicovich-Wilson, C. M.; Pascale, F.; Civalleri, B.; Doll, K.; Harrison, N. M.; Bush, I. J.; D'Arco, P.; Llunell, M. CRYSTAL 2009 User's Manual; University of Torino, Torino, 2009.

[53] Doll, K. Comput. Phys. Comm. 2001, 137, 74-88.

[54] Doll, K.; Harrison, N. M.; Saunders, V. R. Int. J. Quantum Chem. 2001, 82, 1-13. 
[55] Civalleri, B.; D’Arco, P.; Orlando, R.; Saunders, V. R.; Dovesi, R. Chem. Phys. Lett. 2001, 348, 131.

[56] Broyden, C. G. J. I. Math. Appl. 1970, 6, 76-90.

[57] Fletcher, R. Comput. J. 1970, 13, 317-322.

[58] Goldfarb, D. Math. Comput. 1970, 24, 23-26.

[59] Shanno, D. F. Math. Comput. 1970, 24, 647-656.

[60] Ferrero, M.; Rérat, M.; Orlando, R.; Dovesi, R. Coupled Perturbed HartreeFock Calculation of the static polarizability for periodic systems: implementation in the CRYSTAL code, Vol. Computation in Modern Science and Engineering, Volume 2B of AIP Conference Proceedings; T. E. Simos and G. Maroulis, American Institute of Physics, 2007.

[61] Ferrero, M.; Civalleri, B.; Rérat, M.; Orlando, R.; Dovesi, R. J. Chem. Phys. 2009, 131, 214704.

[62] Jmol 3d engine. http://jmol.sourceforge.net.

[63] Animation of vibrational normal modes on a web page using jmol. http://www. theochem. unito.it/crystal_tuto/mssc2008_cd/tutorials/webvib/index.html.

[64] Inkscape program. http://www. inkscape.org.

[65] Decius, J.; Hexter, R. Molecular vibrations in crystals; McGraw-Hill, 1977.

[66] Born, M.; Huang, K. Dynamical theory of crystal lattices; Oxford University Press: Oxford, 1954.

[67] Gonze, X.; Lee, C. Phys. Rev. B 1997, 55, 10355.

[68] Kleinmann, D. A.; Spitzer, W. G. Phys. Rev. 1962, 125, 16.

[69] Hazen, R. Am. Mineral. 1976, 61, 1280-1293.

[70] Prencipe, M.; Noël, Y.; Bruno, M.; Dovesi, R. Am. Mineral. 2009, 94, 986-994. 
[71] Prencipe, M.; Nöel, Y.; Civalleri, B.; Roetti, C.; Dovesi, R. Phys. Chem. Min. 2006, 33, 519-532.

[72] Pascale, F.; Tosoni, S.; Zicovich-Wilson, C.; Ugliengo, P.; Orlando, R.; Dovesi, R. Chem. Phys. Lett. 2004, 396, 308-315.

[73] Shannon, R. D.; Subramanian, M. A. Phys. Chem. Min. 1989, 16, 747-751.

[74] Deer, W. A.; Howie, R. A.; Zussman, J. Rock forming minerals, second edition, Vol. 1A; Longman: London, 1982. 